\title{
Spatial and temporal fluctuations in sunspots derived from MDI data
}

\author{
S. K. Solanki ${ }^{1}$ and I. Rüedi ${ }^{2}$ \\ 1 Max-Planck-Institut für Aeronomie, Max-Planck-Str. 2, 37191 Katlenburg-Lindau, Germany \\ 2 Physikalisch-Meteorologisches Observatorium Davos/World Radiation Center, Dorfstr. 33, 7260 Davos-Dorf, Switzerland
}

Received 23 April 2003 / Accepted 15 July 2003

\begin{abstract}
The penumbra radiates an energy flux that is 0.75-0.8 times the photospheric value. One mechanism proposed to bring this flux to the surface is interchange convection according to which hot flux tubes rise to the surface, lie horizontally there while they cool and finally sink down again. We search for possible signatures of such a process using time series of magnetograms and continuum images recorded by the Michelson Doppler Imager (MDI) in its high resolution mode (0.6" pixels). The data reveal that at the spatial scales accessible to MDI, magnetic structures are on average smaller in the azimuthal direction than brightness features. The small-scale magnetic pattern resolvable by MDI lives for well over two hours, i.e. longer than the brightness pattern. As shown in a parallel paper (Schlichenmaier \& Solanki 2003) this result, taken together with theoretical predictions, suggests that interchange convection is unable to account for the observed penumbral radiative flux. The need for higher resolution data obtained under stable conditions is pointed out.
\end{abstract}

Key words. Sun: activity - Sun: magnetic fields - Sun: photosphere - sunspots

\section{Introduction}

Sunspots harbor considerable fine structure (notably in the penumbra) which changes with time. The investigation of spatial variations and temporal fluctuations in sunspots using ground-based observations is sensitive to the inhomogeneous and variable seeing caused by turbulence in the Earth's atmosphere. In spite of the sometimes excellent spatial resolution achieved by such observations (e.g. Scharmer et al. 2002) some uncertainty remains when investigating time series, since that needs a time-independent resolution. This problem is particularly acute when considering magnetograms which require longer integration times to record.

In the present paper data recorded with the Michelson Doppler Imager (MDI, Scherrer et al. 1995) on the Solar and Heliospheric Observatory ( $\mathrm{SOHO}$ ) is used to investigate the temporal and spatial fluctuations present in sunspots and compare them with sunspot models. These data have the advantage of being unaffected by seeing fluctuations and scattered light in the Earth's atmosphere. Although the moderate spatial resolution of the instrument ( $0.6^{\prime \prime}$ per pixel) is a significant disadvantage, the fact that it is well defined, time-independent and homogeneous provides some compensation for this, in particular for evolution studies. Hence, in spite of the fact that MDI data may miss a considerable fraction of the structure at the finest

Send offprint requests to: $\mathrm{S}$. K. Solanki, e-mail: solanki@linmpi.mpg.de scales, we feel that we can trust the results obtained for the remainder.

In the present paper we employ MDI data to investigate the structure and evolution of the thermal (continuum brightness) and magnetic (magnetogram signal) fine structure of sunspots. In a separate paper (Schlichenmaier \& Solanki 2003, hereafter Paper II) we compare the results of the analysis with the expectations from theory. This allows us to set constraints on how the penumbra is heated. A similar analysis needs to be repeated with higher spatial resolution data of comparable quality. Such data are expected to become available from the Solar-B mission (Shimizu et al. 2002) and at even higher resolution from the balloon borne Sunrise telescope (Schmidt et al. 2001; Solanki et al. 2002).

The structure of this paper is as follows: in Sect. 2 we present the observations. Section 3 addresses the temporal variations of the intensity and the magnetic field observed throughout the sunspot. The spatial variations are discussed in Sect. 4. Spatial and temporal fluctuations are compared with each other in Sect. 5. Finally, our conclusions are presented in Sect. 6.

\section{Observational data and analysis procedure}

The observations consist of MDI continuum intensity and magnetogram timeseries. We investigated a total of four large sunspots which, however, all gave very similar results. In this paper we therefore concentrate on the description of the results obtained in one representative sunspot. This sunspot was chosen since it showed little overall evolution over a duration 

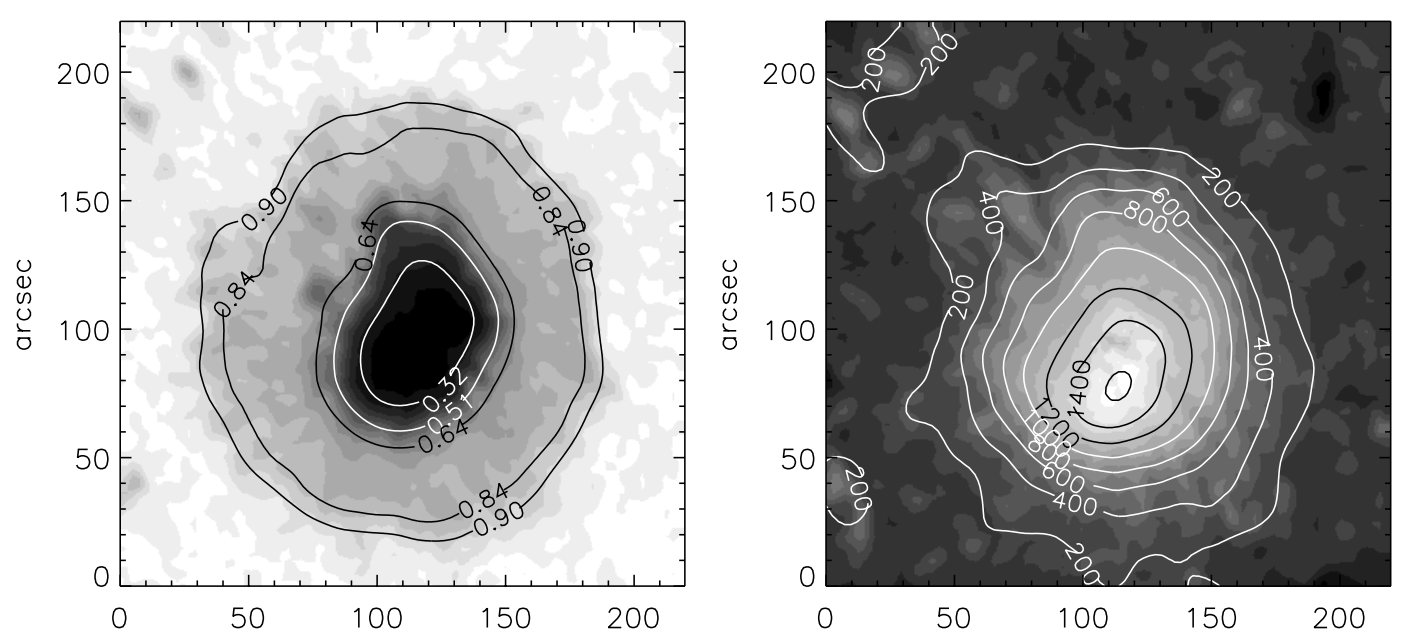

Fig. 1. Paths or contours of constant smoothed intensity and magnetogram signal overplotted on an intensity image (left) and a magnetogram of the sunspot (right), respectively. The intensity values are normalized to the average intensity of the quiet Sun, while the magnetogram signal is given in Gauss. The intensity and magnetogram contours are similar but not identical in shape, partly because the sunspot was not located exactly at sun centre. The inner most contour on the magnetogram corresponds to $1600 \mathrm{G}$. The contours have been smoothed as described in the text.

longer than the temporal interval analysed here and also because it was the most symmetric sunspot in our sample. The analysed data were recorded on 19th June, 1998 in the active region NOAA 8243 located close to the central meridian ( $\mu=\cos \theta=0.94$, where $\theta$ is the heliocentric angle). MDI was operated in its high-resolution mode, i.e. with a pixel size of $0.605^{\prime \prime}$. Magnetograms and intensity images were obtained at a cadence of 1 per minute. Two observing sequences are analysed here. Their lengths are 80 and $200 \mathrm{~min}$, respectively. They are taken from a single, much longer time series. They were chosen so as to have as little evolution of the sunspot during the investigated series. The reason to restrict ourselves to these relatively short time series is to avoid contaminating the results by sunspot evolution, which, although weak, is not altogether absent. Other fragments of the series showed similar results. Solar rotation was compensated prior to the analysis, so that in the reduced timeseries, each pixel follows the same point on the solar surface. The analysis of the $200 \mathrm{~min}$ timeseries is discussed in Sects. 3 and 4, while the 80-min timeseries is discussed in Sect. 5.

We concentrate here on the small-scale spatial fluctuations and their temporal variation as seen in both the intensity and magnetogram signal throughout the sunspot. For this purpose we first need to separate out the large-scale spatial variations and their evolution. Hence we determine smooth contours of constant intensity and magnetogram signal in the sunspot. The time series are first temporally averaged and then smoothed with a box-car function having a width of 9 pixels, in order to suppress the fine structure of the sunspot. The influence of box-cars with smaller and larger widths was also investigated. The exact amount of smoothing turned out to have little influence on the results. In the temporally averaged and smoothed images, intensity and magnetogram signal contours are determined. The analysis of the non-averaged and non-smoothed data is then carried out along those contours. Any fluctuation seen in the data along such a contour is due to variations on a scale smaller than the box-car. This manner of choosing contours or paths has the advantage of applicability also to less symmetric sunspots than the circular paths employed previously by, e.g., Title et al. (1993) and Lites et al. (1993). Since no sunspot is exactly circular, we also expect this technique to be in general more accurate in isolating small-scale fluctuations. Figure 1 shows the intensity and magnetic field paths plotted onto an intensity and magnetogram image, respectively. Note the departures from a circular shape in this figure. If we subtract the spatially smoothed 5-min averaged images from the untreated 5-min averaged image (5-min averages were used in order to reduce the noise) the large scale changes in intensity and magnetogram signal dominating Fig. 1 are removed, so that the small scale structure becomes better visible (see Fig. 2). In continuum intensity the umbral and penumbral boundaries are most prominently visible, where evolution of the sunspot's shape produces strong contrasts. In the magnetogram signal, however, radial structures dominate the image.

In the following we present and discuss in particular the analysis of intensity contours at levels $0.32 I_{\mathrm{c}}$ (umbra), $0.51 I_{\mathrm{c}}$ (transition umbra-penumbra), $0.64 I_{\mathrm{c}}$ (inner penumbra) and $0.84 I_{\mathrm{c}}$ (outer penumbra) as well as the magnetogram levels at $400 \mathrm{G}$ (outer penumbra), $800 \mathrm{G}$ (inner penumbra), $1200 \mathrm{G}$ (transition umbra-penumbra) and $1600 \mathrm{G}$ (umbra). The longitudinal field strengths given here are those obtained directly from MDI using the standard calibration procedure. They are too small, compared with literature values (see Solanki 2003 for a compilation), in particular in the umbra. This points to uncertainties in the deduced longitudinal field values in cool atmospheres, in agreement with the findings of the MDI team (R. Bush and N. Meunier, private communication). This uncertainty should have little influence on the results of the present analysis, except possibly to underestimate the absolute magnitude of the magnetic fluctuations in the umbra, although the relative magnitude is expected to be unaffected. 

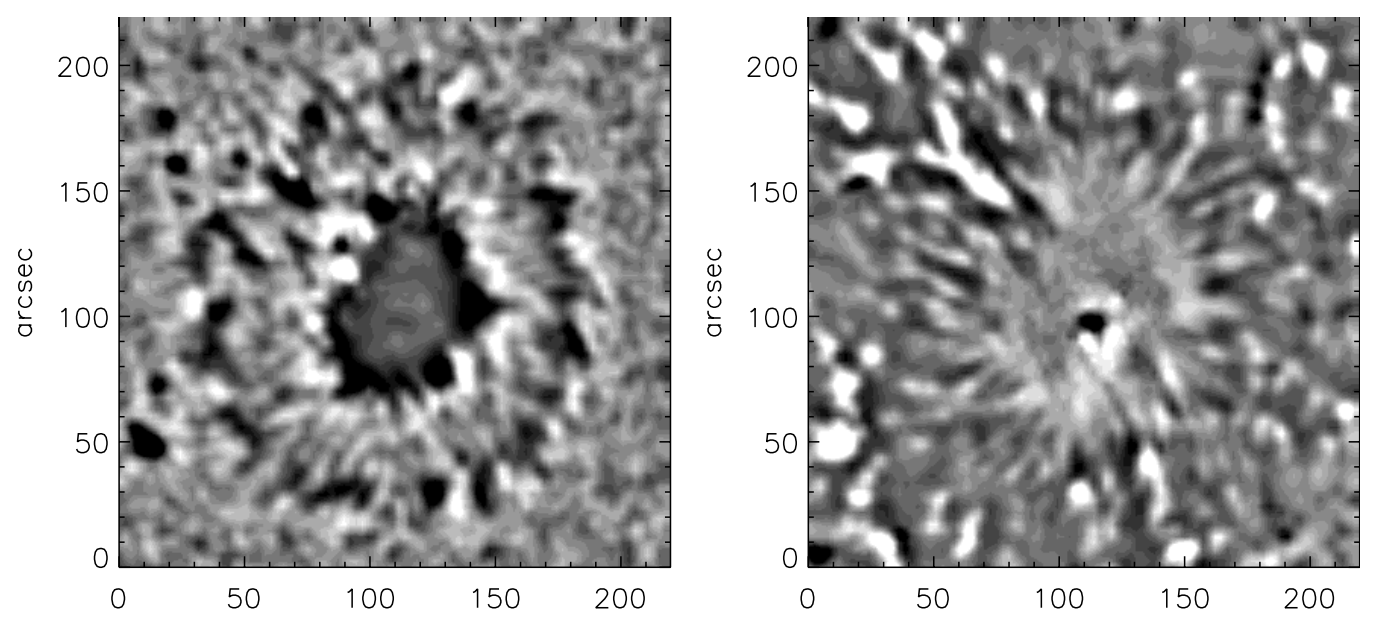

Fig. 2. Residuals after subtracting a spatially smoothed and 5 minute averaged image from the original 5 min averaged image. Left: residuals of the intensity, right: residuals of the magnetogram.

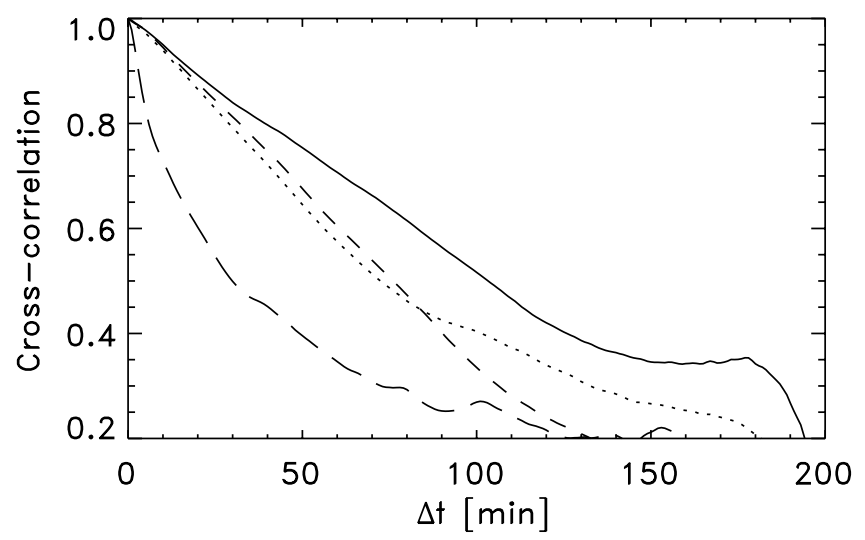

Fig. 3. Cross-correlation coefficient of the intensity $I$ at different times as a function of time-interval. The four curves correspond to different contour levels: $I=0.32 I_{\mathrm{c}}$ (solid), $I=0.51 I_{\mathrm{c}}$ (dashed), $I=0.64 I_{\mathrm{c}}$ (dotted) and $I=0.84 I_{\mathrm{c}}$ (long dashed). Note that the statistics become increasingly poorer for lags approaching the length of the time series and the plotted curves increasingly unreliable.

\section{Temporal variations}

\subsection{Intensity structures}

In this section we investigate the lifetime of the intensity structures. It is determined by computing the cross-correlation function between the intensity values along a given contour at different times. For that purpose we consider the time series lasting $200 \mathrm{~min}$. We determine the cross-correlation of the intensity along the path at a time $t$ with the intensity along the same path at every later time $t+\Delta t$ and sort them according to $\Delta t$. Finally, in order to reduce the noise level and random fluctuations in the cross-correlation function, we compute the average cross-correlation of all the profile pairs corresponding to the same $\Delta t$. Prior to averaging, the various cross-correlations with the same $\Delta t$ are shifted such that their maxima overlap. In this way the signal-to-noise ratio is increased while avoiding a reduction of the cross-correlation because of pattern shifts in azimuthal direction with time. Figure 3 shows the maximum values of the cross-correlation functions vs. $\Delta t$ for the
Table 1. Lifetime estimates of small-scale brightness and magnetic structures.

\begin{tabular}{lcccc}
\hline \hline Contour $I_{\mathrm{c} s p} / I_{\mathrm{c} 9 \mathrm{~s}}$ & 0.32 & 0.51 & 0.64 & 0.84 \\
\hline Lifetime $(\mathrm{min})$ & 104 & 75 & 72 & 28 \\
\hline \hline Contour $\langle B \cos \gamma\rangle(\mathrm{G})$ & 1600 & 1200 & 800 & 400 \\
\hline Min. lifetime $(\mathrm{min})$ & 73 & 110 & 74 & 115 \\
Corr. lifetime (min) & $256^{\mathrm{a}}$ & 138 & 80 & 119 \\
\hline
\end{tabular}

${ }^{a}$ Estimated from linear extrapolation.

intensity contour levels of value $0.32 I_{\mathrm{c}}, 0.51 I_{\mathrm{c}}, 0.64 I_{\mathrm{c}}$ and $0.84 I_{\mathrm{c}}$ (cf. Fig. 1). From the umbra through the inner penumbra to the outer penumbra, the stability of the intensity structure decreases. In particular in the outer penumbra the maximum of the cross-correlation initially drops very rapidly, pointing at strong variations of the intensity structure over relatively short time-scales. For longer lags fewer cross-correlation functions are available for averaging, so that the information content of the curves decreases rapidly and they become increasingly unreliable.

If we take the lifetime of the brightness pattern to be the point at which the cross-correlation drops below 0.5 , we obtain lifetimes of small-scale features (or patterns) as given in Table 1 (the first two rows refer to intensity structures, which we deal with in this section). Note that a correlation of 0.5 is still quite significant and thus this definition probably underestimates the true lifetimes. $I_{\mathrm{c}}^{\mathrm{sp}}$ refers to the continuum intensity of the sunspot, $I_{\mathrm{c}}^{\mathrm{qs}}$ to that of the quiet Sun.

\subsection{Magnetic structures}

In Fig. 4 we plot the maximum of the cross-correlation between the magnetogram signal at times $t$ and $t+\Delta t$ along the smoothed magnetogram contour levels (at $400 \mathrm{G}, 800 \mathrm{G}, 1200 \mathrm{G}$ and $1600 \mathrm{G})$. The cross-correlations are averaged as described in Sect. 3.1 before their maxima are determined. The sharp drop present at $\Delta t=1 \mathrm{~min}$, corresponding to a single time-step, for 


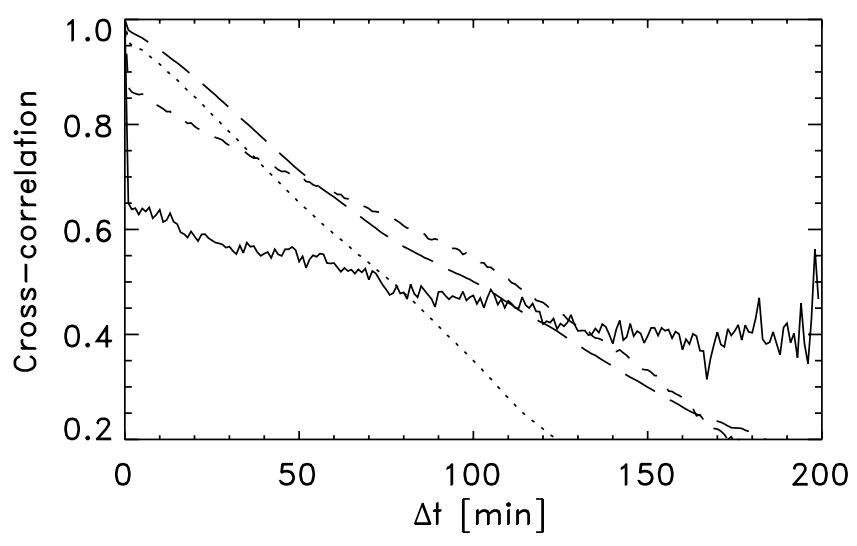

Fig. 4. Cross-correlation coefficient of the magnetogram signal as a function of time-lag $\Delta t$ for contour levels at different magnetogram signals: $B=1600 \mathrm{G}$ (solid), $B=1200 \mathrm{G}$ (dashed), $B=800 \mathrm{G}$ (dotted) and $B=400 \mathrm{G}$ (long dashed).

the solid $(1600 \mathrm{G})$ and dashed $(1200 \mathrm{G})$ curves is due to the significant noise present in the magnetogram signal in the umbra and at the transition to the penumbra. The enhanced noise is basically due to poor photon statistics in the dark parts of sunspots combined with the fact that the magnetogram signal is much weaker than the intensity. Because noise is uncorrelated it lowers the correlation by an amount that is independent of $\Delta t$. Due to this effect, which is also present in weakened form at contours $400 \mathrm{G}$ and $800 \mathrm{G}$, the lifetimes estimated directly from the curves plotted in Fig. 4 are lower limits, in particular for the umbral values. These values are listed in the second last line of Table 1 (denoted as minimum lifetimes).

We feel that a better estimate is obtained by neglecting the jump in cross-correlation during the first minute caused by the noise. We extrapolate each cross-correlation curve back to time-lag 0 and then shift the curve up so that this extrapolated value becomes unity. Any error in estimating the magnitude of this initial drop due to noise is converted almost linearly into an error in the enhancement of the lifetime obtained by this procedure. This translates into increasingly large errors toward higher fields. In the case of the $1600 \mathrm{G}$ contour, assuming a generous $10 \%$ error in determining the initial drop, we also obtain a $10 \%$ error in the total lifetime introduced by the extrapolation and the shifting of the curves. The lifetimes obtained in this manner are listed in the last row of Table 1 (corrected lifetimes). They are significantly larger than the lifetimes of the intensity structures. In the case of the outer penumbral structures (long-dashed curves) the magnetic pattern lives four times longer than the intensity pattern.

The lifetime (both minimum and corrected) of magnetic structures at the $800 \mathrm{G}$ contour is somewhat smaller than at $1200 \mathrm{G}$ and $400 \mathrm{G}$. This can also be seen in Fig. 4 where the cross-correlation curve of the $800 \mathrm{G}$ contour drops more rapidly than the others. We could find no instrumental effect that could cause this reduction (e.g. if residual instrumental cross-talk from Stokes $I$ to $V$ were to diminish the apparent lifetime of magnetic structures, then we would expect the $400 \mathrm{G}$ contour to suffer more, since the lifetime of intensity structures in the outer penumbra is considerably lower than elsewhere in the sunspot; see Table 1). The reduced lifetime at the $800 \mathrm{G}$ contour could well be due to the fact that this curve is close to the umbra/penumbra boundary and is therefore more sensitive to the evolution of the sunspot than other curves.

Our results are in good agreement with those of Lites et al. (1993), who found no significant change in the magnetic structure of a sunspot over a period of $30 \mathrm{~min}$.

\section{Spatial variations}

\subsection{Intensity structures}

The small-scale spatial variations of the brightness along smoothed iso-contours are studied with the help of autocorrelation functions. At each time step the auto-correlation along the given contour is calculated and then averaged over the different time steps. Note that changes in the spatial pattern do not influence any significant peaks in the auto-correlation as long as the spatial scales are time-independent. These averaged auto-correlations are plotted in Fig. 5 for four brightness levels (the same as discussed in Sect. 3.1). The width of the central peak is a measure of the spatial scale of brightness structures. We quantify it by the full width at half maximum ( $F W H M$; i.e. the full width at auto-correlation 0.5). The FWHM lies between 2.3 and 3.6 pixels (corresponding to 1.4 arcsec and 2.2 arcsec, respectively), irrespective of the location within the sunspot. This value is larger than the spatial resolution $(\approx 2$ pixels) and suggests that at least some of the fine structure is already resolved by MDI, although observations with higher spatial resolution show considerably smaller scales as well. The size scales revealed by the auto-correlations may be related to the fact that although the smallest scales are most striking to the eye (e.g. penumbral filaments, umbral dots) larger scale fluctuations are rather strong both in the umbra and the penumbra and may even dominate (Grossmann-Doerth \& Schmidt 1981; Collados et al. 1987; Sobotka et al. 1993).

Another interesting feature of the auto-correlations is the presence of sidebands in the penumbra, most prominently in the inner penumbra. The sidebands are indicative of a measure of periodicity at a scale of 6-9".

\subsection{Magnetic structures}

Auto-correlation functions have also been determined for the magnetic field along the relevant contour lines. Averages of these auto-correlation functions are plotted in Fig. 6. The central peak in this case again has a similar width at all studied values of the large-scale magnetic field, which implies that the size of magnetic structures (e.g. the width of magnetic filaments in the penumbra) is practically independent of position in the sunspot. The $F W H M$ of the autocorrelation function is approximately 2 pixels ( 1.2 arcsec) along almost all magnetic contours, rising to 2.6 pixels $(1.6$ arcsec) only at the outer edge of the penumbra. This means that the magnetic structure loses much of its coherence within the spatial resolution of MDI, which may be partly due to the large noise in the magnetograms (see Fig. 4 and Sect. 3.2). However, this cannot be the only reason for the narrower peaks in Fig. 6 compared to Fig. 5. In Fig. 4 

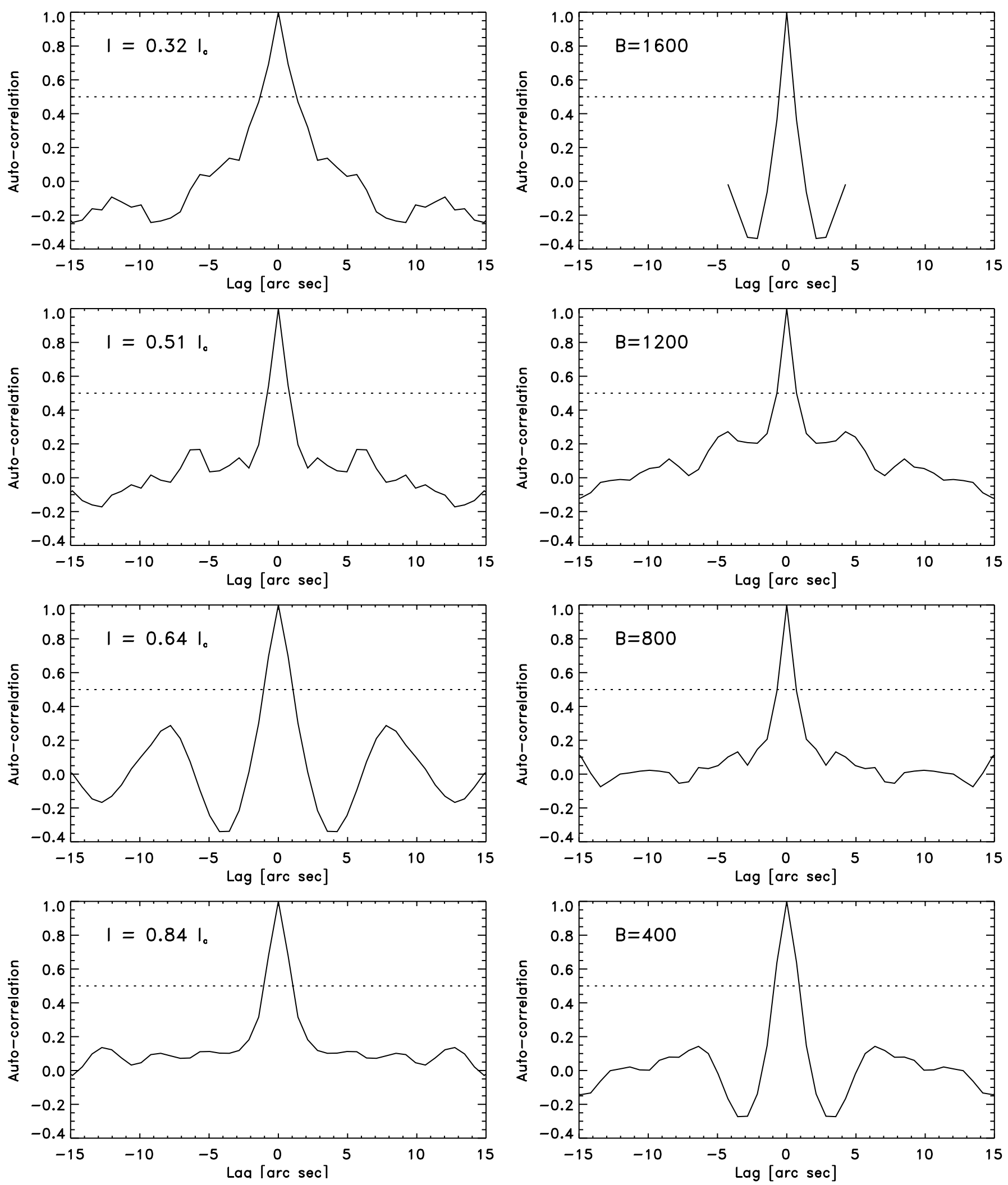

Fig. 5. Average spatial auto-correlation coefficient of the intensity as a function of the spatial lag, for different average intensity levels as indicated in each panel.

Fig. 6. Average spatial auto-correlation coefficient of the magnetogram signal as a function of the spatial lag, for different average magnetogram signal levels as indicated in each panel.

the cross-correlation drops by less than 0.35 due to noise everywhere in the spot. In the penumbra this noise-induced drop is less than 0.15 . Compared with the very rapid and large decrease

of the autocorrelation function within a lag of an arcsec seen in Fig. 6, the drop due to noise is minute there. We therefore 
expect that the difference in width of the intensity and magnetic autocorrelation functions is real.

A narrower central peak of the autocorrelation function implies structure at a smaller scale. Hence, on the spatial scales accessible to MDI Figs. 5 and 6 suggest that the dominant spatial scale on which brightness structure is ordered is somewhat larger than the corresponding scale for the magnetogram signal. Furthermore, the $F W H M$ of the autocorrelation function of the magnetogram signal is as low as 2 pixels, i.e., the autocorrelation drops from 1 to 0.5 for a lag of a single pixel. This implies that the magnetic pattern changes significantly from one pixel to the next, so that considerable magnetic fine structure is present at or below the spatial resolution of MDI, as is well known from the literature (see Solanki 2003 for a review).

Side bands are most prominent in the outermost part of the penumbra, in contrast to the intensity. This may be due to the fact that the magnetic fine-structure is more prominent there (at the spatial resolution accessible to MDI) or due to the increased visibility of magnetic fine-scale structure.

We stress that the fluctuations studied here are those of the magnetogram signal which may have different sources, ranging from field-strength variations to variations in the thermodynamic quantities (which can influence the magnetogram signal through changes in the line profiles), although the most likely is field inclination variations, which have been detected or modelled by numerous previous investigators (e.g., Degenhardt \& Wiehr 1991; Schmidt et al. 1992; Title et al. 1993; Solanki \& Montavon 1993; Martínez Pillet 2000). When only the longitudinal component of the field is measured then such fluctuations are best visible for an almost transverse magnetic field (e.g., Ploner \& Solanki 1997), i.e. near the outer edge of the penumbra for a sunspot observed near solar disc centre. This may also contribute to the presence of sidelobes at the outermost contour.

The effect of increased visibility of magnetic inclination fluctuations near the outer boundary of the sunspot may be the dominant source of the increased side band structure, since observations with higher spatial resolution and/or sampling the whole Stokes vector do not indicate strongly increasing fluctuations towards the sunspot's boundary. For example, Lites et al. (1993) find the magnitude of magnetic inclination fluctuations to be almost constant throughout the sunspot and to increase rapidly only near the outer penumbral boundary. They also find that field strength fluctuations are largest at the umbral boundary, possibly due to the fact that these authors determine fluctuations along circular paths, whereas the umbral boundary is serrated. The amplitude of the magnetic inclination angle variations deduced by Title et al. (1993) are seen to be approximately independent of radial distance in the penumbra (their Fig. 9). From their Fig. 8 the typical scale of the fluctuations with large amplitude is $1-5^{\prime \prime}$, i.e. in a range that can (just) be resolved by MDI in its high resolution mode.

Note that the technique used here, the formation of residuals (similar to high-pass filtering) and the computation of autocorrelation functions, reveals the presence and, to a certain extent, the size scales of fine structure. It does not, however, provide information on the amplitude of the fine structure. The amplitudes of the spatial and temporal fluctuations are discussed in the following section.

\section{Comparison of temporal and spatial fluctuations}

The spatial and temporal sampling of MDI are appropriate relative to each other in the sense that the time required by a sound wave to cross one pixel of MDI is approximately $1 \mathrm{~min}$, which is the temporal sampling interval. Therefore it is appropriate to compare how spatial and temporal variability depend on position in the sunspot, whereby the standard deviation is used as a measure of variability.

In the case of spatial fluctuations we have determined the standard deviation of the intensity, $\sigma_{\mathrm{s}}(I)$, along 8 contour lines of intensity, and similarly the standard deviation of the magnetogram signal, $\sigma_{\mathrm{s}}(B)$, along contours of line-of-sight magnetic field. The standard deviation values thus obtained for each time step are then averaged over the whole time series and represented by the stars in Fig. 7. Note that in Fig. 7 the umbra is represented by the points at the left (low intensities and high field strengths). Note, however, that the horizontal scales of the two figures do not correspond exactly to each other since the intensity and magnetogram signal exhibit different spatial variations. The error bars represent the standard deviation of the distribution of the $\sigma_{\mathrm{s}}(I)$ and $\sigma_{\mathrm{s}}(B)$ values, respectively, at a given contour level.

The temporal fluctuations are estimated by taking a given pixel along a contour and following it over time. The results are then averaged over all the spatial pixels along an iso-contour, giving $\sigma_{\mathrm{t}}(I)$ and $\sigma_{\mathrm{t}}(B)$, which are plotted in Fig. 7 (diamonds).

Such a procedure is imperfect, since spatial drifts due to the evolution of the sunspot, which are always present to some degree, do influence the results. Therefore, we restrict this part of the analysis to a 80-min long timeseries. Hence a part of the standard deviation of the time series is probably cross-talk from the spatial variation. Because the spatial structures lose coherence on particularly small spatial scales in the magnetogram, we expect this cross-talk to be more severe for the magnetic time series (Fig. 7b).

According to Fig. 7a $\sigma_{\mathrm{s}}(I)$ and $\sigma_{\mathrm{t}}(I)$ peak at an $I$ of around $0.5 I_{\mathrm{c}}$, which corresponds to the boundary between the umbra and penumbra. The large fluctuations there are due to the strong brightness gradient, the raggedness of the umbral boundary and its evolution. Large $\sigma_{\mathrm{s}}(I)$ and $\sigma_{\mathrm{t}}(I)$ are also reached at the outer penumbral boundary. This may again partly be due to its raggedness and evolution (compare with Fig. 2). The higher $\sigma_{\mathrm{s}}(I)$ and $\sigma_{\mathrm{t}}(I)$ values in the penumbra are, however, expected to be largely due to the penumbral filaments. Note that the slow drop of $\sigma_{\mathrm{s}}(I)$ in Fig. 7 from its peak at a relative intensity of around 0.51 towards both higher and lower values of $I$ extends over only a small part of the sunspot. A glance at Fig. 1 reveals that the relative intensity contours between 0.32 and 0.64 lie rather close together and delineate the umbra-penumbra boundary. The true, intrinsic variability of the umbra is thus only represented by the point at 0.22 , which shows by far the lowest $\sigma_{\mathrm{s}}(I)$ and $\sigma_{\mathrm{t}}(I)$ throughout the sunspot. This is in agreement with the general impression that the penumbra exhibits more fine structure that the umbra.

Quite clearly the temporal variability is smaller than the spatial variability at all intensity levels. Figure 7a confirms what can be deduced already by comparing Figs. 3 and 5 . 

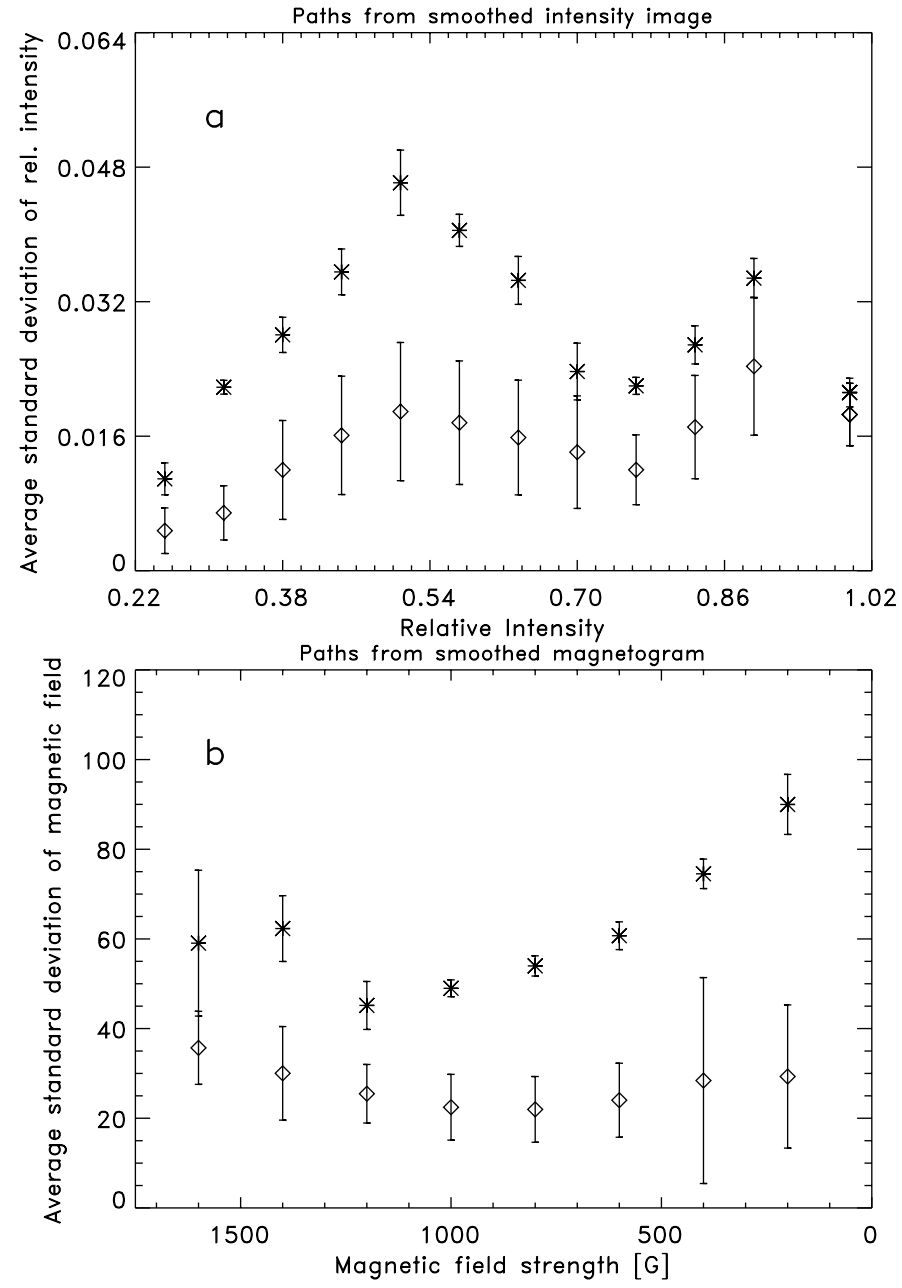

Fig. 7. a) Average standard deviation of the intensity and b) of the magnetogram signal along the intensity and magnetogram contours as a function of the relative intensity and magnetic field strength, respectively. Stars: spatial variations, diamonds: temporal variations.

In the former case the cross-correlation has in most cases not yet dropped to the 0.5 level within $30 \mathrm{~min}$ (i.e. 30 temporal pixels), while it drops (often even below zero) within a few pixels in the spatial direction. Note, however, that $\sigma_{\mathrm{s}}(I)$ and $\sigma_{\mathrm{t}}(I)$ also include uncorrelated variations and thus provides information independently of the cross-correlation functions.

The largest spatial fluctuations of the magnetogram signal (Fig. 7b) are found near the outer penumbral boundary (cf. Fig. 6). The small increase of $\sigma_{\mathrm{s}}(B)$ and $\sigma_{\mathrm{t}}(B)$ in the umbra, at the largest $B$ values, is probably due to the increased noise in the signal, which also causes the prominent drops within one pixel of the corresponding cross-correlation functions in Fig. 4, which are significantly smaller for magnetogram values typical of the penumbra. Leaving these points in Fig. $7 \mathrm{~b}$ aside, a steady, nearly quadratic increase of the spatial fluctuations towards the outer boundary is evident. This figure shows even more clearly than Fig. 6 the sharp increase of the fine-scale structure in the magnetogram towards the outer penumbra. Possible reasons for an increased visibility of penumbral magnetic fine-structure are given in Sect. 4.2. The umbral boundary is in no way prominent in $\sigma_{\mathrm{t}}(B)$ or $\sigma_{\mathrm{s}}(B)$, suggesting that there is very little cross-talk of relevance for our analysis from $I$ to the magnetogram. In contrast to $\sigma_{\mathrm{s}}(B)$ the temporal fluctuations, $\sigma_{\mathrm{t}}(B)$ are practically independent of $B$, except for a small increase in the umbra, again probably due to noise.

Hence, as we go out from the centre of the sunspot towards its edge the ratio of the spatial to temporal variations increases, reaching values as large as a factor of 3. Due to the cross-talk from spatial to temporal variations mentioned earlier this ratio should be considered to be a lower limit.

As spatial resolution is increased, we expect the spatial standard deviation to increase rapidly, since MDI does not resolve much of the fine-scale structure in the penumbra (fibrils typically have a width significantly below 1"; Sütterlin 2001; Scharmer et al. 2002). It is unclear, however, whether an increase in spatial resolution also leads to a corresponding increase in $\sigma_{\mathrm{t}}(B)$ or $\sigma_{\mathrm{t}}(I)$.

It will require higher spatial resolution observations that are equally stable in time to resolve this issue. Recent work by Sütterlin (private communication) suggests, however, that at least $\sigma_{t}(I)$ should not be significantly higher if the spatial resolution is increased 4 -fold.

\section{Conclusions}

The fine structure of sunspots has been a subject of intensive investigation (see reviews by Sobotka 1997; Solanki 2003). Most work has been restricted, however, to the intensity structure and to single snapshots. The reason for this restriction lies in the fluctuating seeing affecting ground-based observations. Achieving high resolution in magnetograms, which require longer integration times, or obtaining time series at uniform resolution become challenging using ground-based observations.

Here we have therefore analyzed time series of intensity images and magnetograms recorded in space by the MDI instrument. We have considered both spatial and temporal fluctuations along contours of equal intensity and magnetogram signal (the isocontours refer to scales larger than 6 arcsec).

We find that the small-scale structures have lifetimes ranging from $30 \mathrm{~min}$ (intensity structures in the outer penumbra) to well over two hours (magnetic features). Also, the lifetime of the magnetic pattern is greater than that of the intensity pattern. Similarly, we obtain that the dominant spatial scale (larger than the spatial resolution of 1.2 arcsec) is 1.4-2.2 arcsec for intensity structures and 1.2-1.6 arcsec for magnetic structures. Furthermore, we show that the amplitude of the temporal fluctuations is significantly smaller than that of the spatial fluctuations. Since the sound-crossing time across a pixel is nearly the observing cadence, this implies that disturbances that produce visible changes in the (small-scale) brightness or magnetic structure of a sunspot propagate at well below the sound speed.

The best known convective phenomenon on the Sun is granulation. In the case of granulation energy can be transported extremely efficiently in a quasi-steady state (overturning convection). For energy transport within sunspots, oscillatory convection (Weiss 2002 and references therein) and interchange convection (Schmidt 1991; Jahn \& Schmidt 1994) have been 
proposed. The energy flux transported by these mechanisms is directly related to dynamic, explicitly time-dependent phenomena. Since gas cannot flow across the field lines, fresh flux has to keep appearing at the surface, causing changes in the thermal and magnetic structuring, which is open to observational tests.

The long lifetimes of the magnetic structures pose problems for the model of interchange convection, as originally proposed by Schmidt (1991) and Jahn \& Schmidt (1994). On the basis of the results obtained here we show in Paper II that a simple theoretical estimate gives energy fluxes nearly an order of magnitude smaller than required to explain the penumbra's radiative flux.

We are well aware of the limitations of the MDI data, in particular regarding the spatial resolution, which implies that the conclusion concerning the insufficiency of interchange convection must be considered preliminary. We stress the need for high-resolution (ideally $0.1^{\prime \prime}$ ) spectropolarimetric observations of sunspots over multiple hours unaffected by seeing in order to finally resolve this question.

Acknowledgements. We thank R. Schlichenmaier for helpful discussions as well as carefully reading the manuscript and an anonymous referee for useful comments on the manuscript.

\section{References}

Collados, M., del Toro Iniesta, J. C., \& Vázquez, M. 1987, Sol. Phys., 105,17
Degenhardt, D., \& Wiehr, E. 1991, A\&A, 252, 821

Grossmann-Doerth, U., \& Schmidt, W. 1981, A\&A, 95, 366

Jahn, K., \& Schmidt, H. U. 1994, A\&A, 290, 295

Lites, B. W., Elmore, D. F., Seagraves, P., \& Skumanich, A. P. 1993, ApJ, 418, 928

Martínez Pillet, V. 2000, A\&A, 361, 734

Ploner, S. R. O., \& Solanki, S. K. 1997, A\&A, 325, 1199

Scharmer G. B., Gudiksen, B. V., Kiselman, D., Löfdahl, M. G. \& Rouppe van der Voort, L. H. M. 2002, Nature, 420, 151

Scherrer, P. H., Bogart, R. S., Bush, R. I., et al. 1995, Sol. Phys., 162, 129

Schlichenmaier, R., \& Solanki, S. K. 2003, A\&A, 411, 257 (Paper II)

Schmidt, H. U. 1991, Geophys. Astrophys. Fluid Dyn., 62, 249

Schmidt, W., Solanki, S. K., Lites, B. W., Title, A. M., \& Martínez Pillet, V. 2001, Astron. Nachr., 322, 363

Schmidt, W., Hofmann, A., Balthasar, H., Tarbell, T. D., \& Frank, Z. A. 1992, A\&A, 264, L27

Shimizu, T., \& the Solar B Team 2002, Adv. Space Res., 29, 2009

Sobotka M. 1997, in Advances in Physics of Sunspots, ed. B. Schmieder, J. C. del Toro Iniesta, M. Vázquez, ASP Conf. Ser., 118,155

Sobotka, M., Bonet, J. A., \& Vázquez, M. 1993, ApJ, 415, 832

Solanki, S. K. 2003, A\&ARv, 11, 153

Solanki, S. K., \& Montavon, C. 1993, A\&A, 275, 283

Solanki, S. K., Schüssler, M., Curdt, W., et al. 2002, in Magnetic Coupling in the Solar Atmosphere, ESA SP-505, 27

Sütterlin, P. 2001, A\&A, 374, L21

Title, A. M., Frank, Z. A., Shine, R. A., et al. 1993, ApJ, 403, 780

Weiss, N. O. 2002, Astron. Nachr., 323, 371 